\title{
Metallicity of mono- and multiperiodic $\beta$ Cephei stars
}

\author{
J. Daszyńska-Daszkiewicz and E. Niemczura
}

\author{
Astronomical Institute of Wrocław University, ul. Kopernika 11, 51-622 Wrocław, Poland \\ e-mail: daszynska@astro.uni.wroc.pl
}

Received 5 March 2004 / Accepted 2 December 2004

\begin{abstract}
Analyzing IUE ultraviolet spectra of $\beta$ Cep pulsating stars we noticed that multiperiodic variables have a larger mean metal abundance in the photosphere, $[\mathrm{m} / \mathrm{H}]$, than monoperiodic ones. We apply statistical tests to verify this dichotomy. We obtain that, with a large probability, the multiperiodic $\beta$ Cep stars have greater values of $[\mathrm{m} / \mathrm{H}]$. This result is consistent with the linear non-adiabatic theory of pulsation of early B-type stars.
\end{abstract}

Key words. stars: abundances - stars: variables: general - methods: statistical

\section{Introduction}

The $\beta$ Cephei pulsators are a well-known and well-studied group of early-type pulsating stars. Oscillations in these stars are strictly connected to the metal abundance $Z$, as they are driven by the classical $\kappa$-mechanism operating in the layer of the metal opacity bump $\left(T \approx 2 \times 10^{5} \mathrm{~K}\right)$ caused by the lines of Fe-group elements (Moskalik \& Dziembowski 1992; Dziembowski \& Pamyatnykh 1993; Kiriakidis et al. 1992; Gautschy \& Saio 1993). The size of the instability domain of $\beta$ Cep stars is very sensitive to the heavy element abundance $Z$, and is smaller for lower values of $Z$ (Dziembowski $\&$ Pamyatnykh 1993; Pamyatnykh 1999). Therefore, the information about the metal abundance in $\beta$ Cep stars is of special importance.

In previous works (Daszyńska 2001; Daszyńska et al. 2003) we derived the metallicity parameter in the atmosphere, $[\mathrm{m} / \mathrm{H}]$, and the mean stellar parameters for all $\beta$ Cephei stars monitored by the International Ultraviolet Explorer. We showed that the metallicity values obtained are independent of effective temperature and surface gravity. Additionally, they are not correlated with the stellar rotation and any of the pulsational parameters, like a dominant period or amplitudes of the light and the radial velocity variations.

In the scope of her Ph.D. Thesis, Daszyńska (2001) suggested that, in general, the multiperiodic $\beta$ Cep stars have higher values of $[\mathrm{m} / \mathrm{H}]$ than the monoperiodic ones. We mentioned this result in Daszyńska et al. (2003), and we confirmed it in Niemczura \& Daszyńska-Daszkiewicz (2005, Paper I), where we applied a more accurate method of determination of stellar parameters. Using the results of Paper I, we verify this hypothesis here by means of statistical tests.

In Sect. 2 we review the derived values of $[\mathrm{m} / \mathrm{H}]$. Section 3 contains the test of statistical hypotheses about the equality of two means. In Sect. 4 we discuss the dichotomy of $\beta$ Cep variables with regard to the metallicity. Conclusions are given in Sect. 5.

\section{Metallicity of $\beta$ Cephei variables from UV spectra}

In previous work (Paper I) we obtained the values of metallicity in the atmosphere for 31 field $\beta$ Cep stars and 16 ones belonging to three open clusters: NGC 3293, NGC 4755 and NGC 6231. Our approach consisted of fitting theoretical fluxes of Kurucz (1996) to low resolution IUE spectra, using the method based on the least-squares optimalization algorithm (Bevington 1969). Errors were estimated by the method of bootstrap resampling. For more details about the method we refer the reader to Niemczura (2003).

We analyzed IUE spectra from two calibrations: NEWSIPS and INES. The estimated values of the sample mean, standard deviation and standard error of the mean, for $[\mathrm{m} / \mathrm{H}]$ derived from these two date sets, are

\section{NEWSIPS:}

$\overline{[m / \mathrm{H}]}=-0.134, s_{[m / \mathrm{H}]}=0.201, s_{\overline{[m / \mathrm{H}]}}=0.029$

INES:

$\overline{[m / \mathrm{H}]}=-0.125, s_{[m / \mathrm{H}]}=0.203, s_{\overline{[m / \mathrm{H}]}}=0.030$.

As we have shown in Paper I, these two populations follow a normal distribution. Applying the two sample $t$-test and the one-way ANOVA test, we found that the two means are not significantly different up to the significance level $\alpha=0.83$. Also, these two data sets give the same dichotomy of $\beta$ Cep stars with regard to the value of metallicity: higher $[\mathrm{m} / \mathrm{H}]$ for multiperiodic pulsators and lower $[\mathrm{m} / \mathrm{H}]$ for monoperiodic pulsators. Therefore, from now on, we will focus only on the NEWSIPS results. 
Table 1. The values of $[\mathrm{m} / \mathrm{H}]$ for the field $\beta$ Cep stars.

\begin{tabular}{rccrc}
\hline \hline HD & Name & $P[\mathrm{~d}]$ & {$[m / \mathrm{H}]$} & Multi. \\
\hline 886 & $\gamma$ Peg & 0.15175 & $-0.04 \pm 0.08$ & $\mathrm{~N}$ \\
16582 & $\delta$ Cet & 0.16114 & $-0.24 \pm 0.09$ & $\mathrm{~N}$ \\
29248 & $v$ Eri & 0.17351 & $0.05 \pm 0.09$ & $\mathrm{Y}$ \\
44743 & $\beta$ CMa & 0.25002 & $0.04 \pm 0.10$ & $\mathrm{Y}$ \\
46328 & $\xi^{1}$ CMa & 0.20958 & $-0.33 \pm 0.19$ & $\mathrm{~N}$ \\
50707 & 15 CMa & 0.18456 & $0.05 \pm 0.10$ & $\mathrm{Y}$ \\
52918 & 19 Mon & 0.1912 & $0.15 \pm 0.10$ & $\mathrm{Y}$ \\
56014 & 27 CMa & 0.0918 & $-0.47 \pm 0.18$ & $\mathrm{~N}$ \\
111123 & $\beta$ Cru & 0.19120 & $-0.14 \pm 0.13$ & $\mathrm{Y}$ \\
116658 & $\alpha$ Vir & 0.1738 & $-0.12 \pm 0.08$ & $\mathrm{~N}$ \\
118716 & $\epsilon$ Cen & 0.16961 & $-0.14 \pm 0.10$ & $\mathrm{Y}$ \\
120307 & $\gamma$ Cen & 0.195 & $-0.26 \pm 0.10$ & $\mathrm{Y}$ \\
122451 & $\beta$ Cen & 0.157 & $-0.03 \pm 0.15$ & $\mathrm{Y}$ \\
126341 & $\tau$ Lup & 0.17735 & $-0.39 \pm 0.16$ & $\mathrm{~N}$ \\
129056 & $\alpha$ Lup & 0.25985 & $0.04 \pm 0.10$ & $\mathrm{Y}$ \\
129557 & BU Cir & 0.12755 & $-0.27 \pm 0.11$ & $\mathrm{Y}$ \\
129929 & V836 Cen & 0.14313 & $-0.05 \pm 0.10$ & $\mathrm{Y}$ \\
132058 & $\beta$ Lup & 0.232 & $-0.35 \pm 0.11$ & $\mathrm{Y}$ \\
136298 & $\delta$ Lup & 0.1982 & $-0.25 \pm 0.11$ & $\mathrm{~N}$ \\
144470 & $\omega{ }^{1}$ Sco & 0.067 & $-0.45 \pm 0.15$ & $\mathrm{~N}$ \\
147165 & $\sigma$ Sco & 0.24684 & $-0.20 \pm 0.20$ & $\mathrm{Y}$ \\
157056 & $\theta$ Oph & 0.14053 & $-0.15 \pm 0.12$ & $\mathrm{~N}$ \\
158926 & $\lambda$ Sco & 0.21370 & $-0.21 \pm 0.08$ & $\mathrm{Y}$ \\
160578 & $\kappa$ Sco & 0.19987 & $-0.19 \pm 0.10$ & $\mathrm{Y}$ \\
163472 & V2052 Oph & 0.13989 & $-0.25 \pm 0.16$ & $\mathrm{~N}$ \\
199140 & BW Vul & 0.20104 & $0.07 \pm 0.12$ & $\mathrm{~N}$ \\
203664 & SY Equ & 0.16587 & $-0.01 \pm 0.21$ & $\mathrm{~N}$ \\
205021 & $\beta$ Cep & 0.19049 & $-0.07 \pm 0.10$ & $\mathrm{Y}$ \\
214993 & 12 Lac & 0.19308 & $-0.20 \pm 0.10$ & $\mathrm{Y}$ \\
216916 & 16 Lac & 0.16917 & $-0.13 \pm 0.13$ & $\mathrm{Y}$ \\
& HN Aqr & 0.15218 & $0.21 \pm 0.09$ & $\mathrm{~N}$ \\
\hline & & & &
\end{tabular}

For the purpose of this work, we review the obtained values of $[\mathrm{m} / \mathrm{H}]$. In Table 1 we show the field $\beta$ Cep stars, while in Table 2 we show $\beta$ Cep stars belonging to the three open clusters. The following columns contain the HD number, the star name, the value of the dominant period and the value of $[\mathrm{m} / \mathrm{H}]$. The information about mono- or multiperiodicity at the present knowledge is given in the last column ( $\mathrm{Y}=$ yes, $\mathrm{N}=$ no).

We divide the sample of $\beta$ Cep stars in two groups: monoperiodic $\beta$ Cep stars (a random variable $X$ ) and multiperiodic ones (a random variable $Y$ ). These samples have sizes $n=17$ and $m=30$, respectively. The sample parameters in these two groups are the following

$\bar{x}=-0.252, s_{x}=0.221, s_{\bar{x}}=0.054$,

$\bar{y}=-0.068, s_{y}=0.156, s_{\bar{y}}=0.029$.

The same parameters obtained from Gaussian fitting are

$\bar{x}=-0.258, s_{x}=0.223, s_{\bar{x}}=0.056$,

$\bar{y}=-0.055, s_{y}=0.188, s_{\bar{y}}=0.046$.
Table 2. The values of $[\mathrm{m} / \mathrm{H}]$ for the $\beta$ Cep stars in clusters.

\begin{tabular}{rcccc}
\hline \hline Name & ID & $P[\mathrm{~d}]$ & {$[\mathrm{m} / \mathrm{H}]$} & Multi \\
\hline NGC 3293 & & & & \\
\hline V380 Car & N3293-27 & 0.2273 & $0.06 \pm 0.10$ & $\mathrm{Y}$ \\
V400 Car & N3293-11 & 0.1458 & $-0.10 \pm 0.12$ & $\mathrm{Y}$ \\
V401 Car & N3293-10 & 0.1689 & $-0.14 \pm 0.12$ & $\mathrm{Y}$ \\
V404 Car & N3293-23 & 0.1621 & $0.27 \pm 0.07$ & $\mathrm{Y}$ \\
V405 Car & N3293-14 & 0.1524 & $0.15 \pm 0.11$ & $\mathrm{Y}$ \\
V412 Car & N3293-65 & 0.1135 & $0.03 \pm 0.11$ & $\mathrm{Y}$ \\
\hline NGC 4755 & & & & \\
\hline BW Cru & N4755-F & 0.203 & $-0.31 \pm 0.15$ & $\mathrm{Y}$ \\
& N4755-G & 0.156 & $-0.56 \pm 0.20$ & $\mathrm{~N}$ \\
& N4755-I & 0.179 & $-0.29 \pm 0.10$ & $\mathrm{~N}$ \\
& N4755-I-13 & 0.232 & $-0.49 \pm 0.12$ & $\mathrm{~N}$ \\
& N4755-III-01 & 0.130 & $-0.50 \pm 0.18$ & $\mathrm{~N}$ \\
\hline NGC 6231 & & & & \\
\hline V947 Sco & N6231-110 & 0.1079 & $-0.03 \pm 0.23$ & $\mathrm{Y}$ \\
V920 Sco & N6231-150 & 0.1012 & $-0.21 \pm 0.19$ & $\mathrm{Y}$ \\
V964 Sco & N6231-238 & 0.0878 & $0.05 \pm 0.15$ & $\mathrm{Y}$ \\
V946 Sco & N6231-261 & 0.0988 & $0.01 \pm 0.13$ & $\mathrm{Y}$ \\
& N6231-282 & 0.1193 & $-0.42 \pm 0.16$ & $\mathrm{Y}$ \\
\hline
\end{tabular}

Distributions of monoperiodic and multiperiodic stars as a function of $[m / H]$, together with Gaussian fits and normal curves, are shown in Fig. 1. In the left panel, the distribution of $[m / \mathrm{H}]$ for monoperiodic $\beta$ Cep stars is shown, whereas in the right panel the distribution of $[\mathrm{m} / \mathrm{H}]$ for multiperiodic $\beta$ Cep stars is shown.

Comparing the sample parameters with those derived from Gaussian fitting we can say that they are the same within the error limits. Additionally we checked the normality of these distributions by using the Shapiro-Wilk test. Thus, we may assume that $X$ and $Y$ have independent normal distributions $N\left(\mu_{X}, \sigma_{X}^{2}\right)$ and $N\left(\mu_{Y}, \sigma_{Y}^{2}\right)$, respectively, where $\mu$ and $\sigma^{2}$ are the mean and the variance, respectively.

\section{Statistical tests}

We are interested in comparing the means of the metallicity of the monoperiodic and multiperiodic $\beta$ Cep stars, called the random variables $X$ and $Y$, respectively. Because the sample sizes are small, we have to do this in two steps. Firstly, we have to test whether the variances of these two variables are equal, and then, if we accept this hypothesis, we test the equality of the two means.

\subsection{Test for the variances}

The null hypothesis $H_{0}: \quad \sigma_{X}^{2}=\sigma_{Y}^{2}$ will be tested against the alternative hypothesis $H_{1}: \sigma_{X}^{2} \neq \sigma_{Y}^{2}$. For this purpose we use the statistic

$F=\frac{(n-1) S_{X}^{2} /(n-1) \sigma_{X}^{2}}{(m-1) S_{Y}^{2} /(m-1) \sigma_{Y}^{2}}=\frac{S_{X}^{2}}{S_{Y}^{2}}$ 

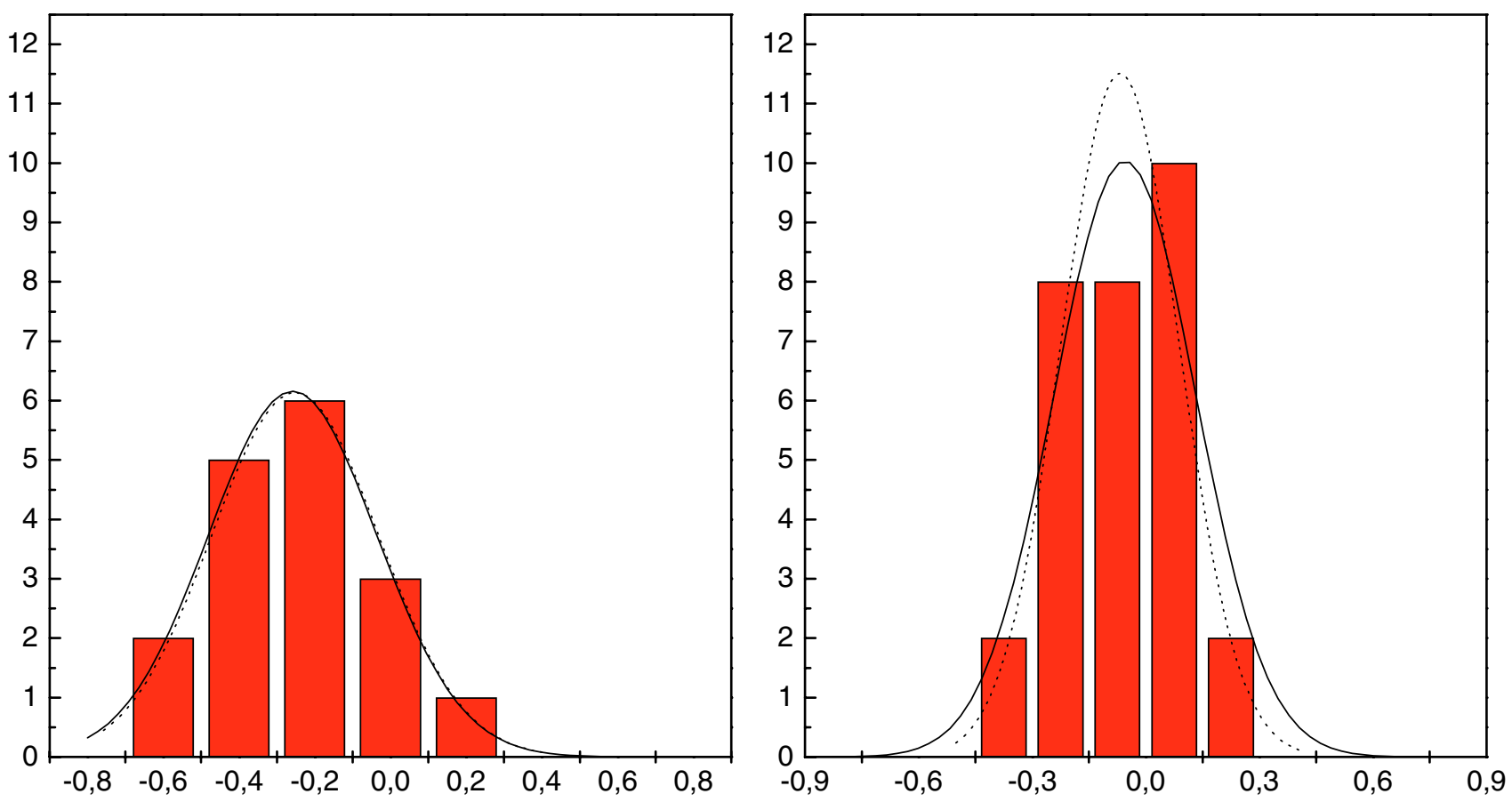

Fig. 1. The distributions of $[\mathrm{m} / \mathrm{H}]$ for monoperiodic $\beta$ Cep stars (left panel) and for multiperiodic ones (right panel). The normal distribution (solid line) and the Gaussian fit (dotted line) are also shown.

which has an $F$-Snedecore distribution with $r_{1}=n-1$ and $r_{2}=m-1$ degrees of freedom. Thus, $F_{\alpha}(n-1, m-1)$ is needed to obtain the $\alpha$ significance level. $S_{X}^{2}$ and $S_{Y}^{2}$ are assumed to be unbiased estimates of the corresponding variances, $\sigma_{X}^{2}$ and $\sigma_{Y}^{2}$, of two independent normal distributions with unknown means. To test the hypothesis $H_{0}: \sigma_{X}^{2}=\sigma_{Y}^{2}$ against a two-sided hypothesis $H_{1}: \sigma_{X}^{2} \neq \sigma_{Y}^{2}$, we assume the critical region given by

$\frac{S_{X}^{2}}{S_{Y}^{2}} \geq F_{\alpha / 2}(n-1, m-1)$

and

$\frac{S_{Y}^{2}}{S_{X}^{2}} \geq F_{\alpha / 2}(m-1, n-1)$.

Taking $s_{x}^{2}$ and $s_{y}^{2}$ as the observed values of unbiased estimates of the variances, we get $F=2.01$. At the significance level $\alpha=0.05$, the critical region, based on the random samples of size $n=17$ and $m=30$, is

$s_{x}^{2} / s_{y}^{2} \geq F_{0.025}(16,29)$ and $s_{y}^{2} / s_{x}^{2} \geq F_{0.025}(29,16)$

or

$F_{0.025}(16,29) \leq s_{x}^{2} / s_{y}^{2} \leq \frac{1}{F_{0.025}(29,16)}=F_{0.975}(16,29)$

hence

$2.30 \leq s_{x}^{2} / s_{y}^{2} \leq 0.39$.

Because our value of $F=2.01$ does not belong to the critical region $(0,0.39>U<2.30, \infty)$, there is no reason to reject the hypothesis $H_{0}: \sigma_{X}^{2}=\sigma_{Y}^{2}$. In this case, the $p$-value, i.e. the smallest level of significance, $\alpha$, at which $H_{0}$ would be rejected, is 0.83 . Thus, with a very high probability we may assume the equality of variances.

\subsection{Test for the means}

Given that the hypothesis of equal variances is accepted, we are ready to test the hypothesis about the means. If random samples of small sizes $n$ and $m$ are taken, the test can be based on the statistic

$T=\frac{\bar{X}-\bar{Y}}{S_{P} \sqrt{1 / n+1 / m}}$,

where

$S_{P}=\sqrt{\frac{(n-1) S_{X}^{2}+(m-1) S_{Y}^{2}}{n+m-2}}$.

The $T$-statistic has a Student's $t$ distribution with $r=n+m-$ 2 degrees of freedom. The critical regions for testing the null hypothesis $H_{0}: \mu_{X}=\mu_{Y}$ against the alternative ones are

(a) $|t| \geq t_{\alpha / 2}(n+m-2)$ if $H_{1}: \mu_{X} \neq \mu_{Y}$,

(b) $t \leq-t_{\alpha}(n+m-2)$ if $H_{2}: \mu_{X}<\mu_{Y}$,

(c) $t \geq t_{\alpha}(n+m-2)$ if $H_{3}: \mu_{X}>\mu_{Y}$.

We recall that the random variable $X$ represents $[m / H]$ for the monoperiodic stars, and the random variable $Y$ represents $[\mathrm{m} / \mathrm{H}]$ for the multiperiodic ones. The case $(a)$ means that we test whether the mean values of $[\mathrm{m} / \mathrm{H}]$ for the monoperiodic $\beta$ Cep stars and the multiperiodic ones are equal or not. In the case $(b)$ we verify the hypothesis of equality of these means against the hypothesis that the mean for monoperiodic variables is less than for the multiperiodic ones. $(c)$ is the test whether the monoperiodic stars have a greater value of $[\mathrm{m} / \mathrm{H}]$ than the multiperiodic ones.

If $\bar{x}, \bar{y}$ and $s_{x}^{2}, s_{x}^{2}$ represent the observed unbiased estimates of the respective parameters $\mu_{X}, \mu_{Y}$ and $\sigma_{X}^{2}=\sigma_{Y}^{2}$ of two independent normal distributions, then the empirical value of the 
test statistic is $t=-3.33$. At $\alpha=0.05$ and $n+m-2=45$ we have the following critical regions for these three alternative hypotheses

(a) $(-\infty,-2.014>\cup<2.014, \infty)$, if $H_{1}: \mu_{X} \neq \mu_{Y}$,

(b) $\left(-\infty,-1.680>\right.$ if $H_{2}: \mu_{X}<\mu_{Y}$,

(c) $<1.680, \infty)$ if $H_{3}: \mu_{X}>\mu_{Y}$.

The values of the observed $t$ belong to the first and the second critical regions. Thus $H_{0}$ is clearly rejected at an $\alpha=0.05$ significance level in favor of the alternative hypothesis $H_{1}$ : $\mu_{X} \neq \mu_{Y}$, as well as in favor of the hypothesis $H_{2}: \mu_{X}<\mu_{Y}$. On the other hand we would not reject the hypothesis $H_{0}$ if the alternative one is $H_{3}: \mu_{X}>\mu_{Y}$. In this case we have to accept that $\mu_{X}=\mu_{Y}$, but it is impossible because $H_{0}$ has already been rejected.

The $p$-value for the two-side hypothesis, $H_{1}: \mu_{X} \neq \mu_{Y}$, is 0.002 , and for the one-side hypothesis, $H_{2}: \mu_{X}<\mu_{Y}$, amounts to 0.001 . Thus, with a large probability, on the basis of these data sets, we can conclude that the mean metallicity for multiperiodic $\beta$ Cep stars is higher than that for monoperiodic ones.

\section{Discussion of the metallicity dichotomy}

The result from our statistical analysis is in agreement with the linear non-adiabatic theory of pulsation of $\beta$ Cep stars. As was shown by Dziembowski \& Pamyatnykh (1993), for greater metal abundance we get more unstable modes. In Fig. 2 we plot the evolution of oscillation frequencies in the Main Sequence phase for four indicated masses and two values of the heavy element abundance $Z$. We considered low degree modes with $\ell=0,1,2$. In these computations we used the WarsawNew Jersey stellar evolution code and the non-adiabatic pulsation code of Dziembowski (1977). We took OPAL opacities and did not include effects of rotation and convective core overshooting. As we can see from Fig. 2, for the higher $Z$ value we get a wider range of unstable frequencies.

Guided by this property, we plot in Fig. 3 two dependencies, taking all studied multiperiodic $\beta$ Cep stars. In the top panel we show the range of the observed frequencies as a function of $[\mathrm{m} / \mathrm{H}]$, whereas in the bottom panel, the range normalized by the sum of the lowest and highest frequency as a function of $[\mathrm{m} / \mathrm{H}]$. In both cases we did not find any correlation. The correlation coefficients, $\tilde{\rho}$, calculated including errors as weights, are equal to -0.31 and -0.45 for the frequency range and the normalized frequency range, respectively.

This result is not surprising. $\beta$ Cep stars are pulsating variables with small amplitudes both in photometry as well as in spectroscopy, and we do not know how many modes we observe amongst the excited ones. The number of known frequencies increases every year. This is mainly thanks to large multisite and multi-technique campaigns for $\beta$ Cep variables. For example, the last campaign of $v$ Eri (Aerts et al. 2004; Handler et al. 2004) gave an increase of the frequency range from 0.14 up to 2.28. The data from the $12 \& 16$ Lac campaign of Handler have been analyzed. Great hopes are pinned also on forthcoming space missions, e.g. the COROT project. Therefore the dependencies in Fig. 3 represent a first trial, which should be constantly updated.
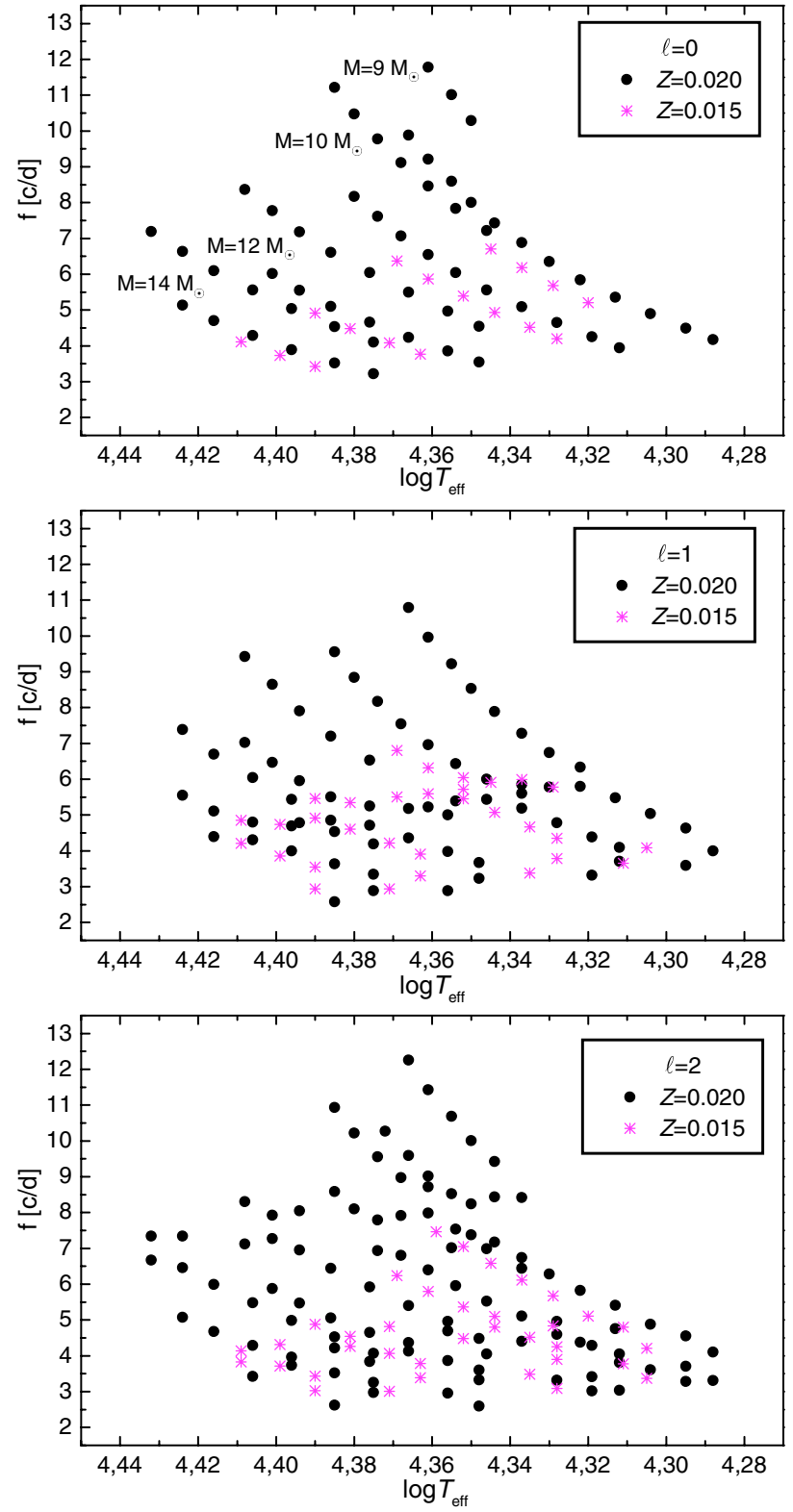

Fig. 2. Frequencies of unstable modes as a function of effective temperature in the MS sequence phase of evolution for four values of mass: $M=9,10,12,14 M_{\odot}$ and two values of the heavy element abundance: $Z=0.020$ and 0.015 . The three panels correspond to three values of the spherical harmonic degree, $\ell$.

\section{Conclusions}

Using statistical tests, we have shown that with a very high probability the multiperiodic $\beta$ Cephei stars have a higher mean metallicity in their atmospheres than the monoperiodic ones. The statistical analysis was based on our last determinations of the metallicity parameter $[\mathrm{m} / \mathrm{H}]$ (Paper I). Finding a normal distribution of $[\mathrm{m} / \mathrm{H}]$ for the mono- and multiperiodic stars, we applied the test of equality of variances of these two samples, represented by $X$ and $Y$ random variables. The test based on the $F$ statistic clearly allowed us to accept the null hypothesis $H_{0}: \sigma_{Y}^{2}=\sigma_{Y}^{2}$. Then we could test the hypothesis of the mean values of metallicity for the mono- and multiperiodic variables. We used the $T$ statistic to define the critical regions for a small 

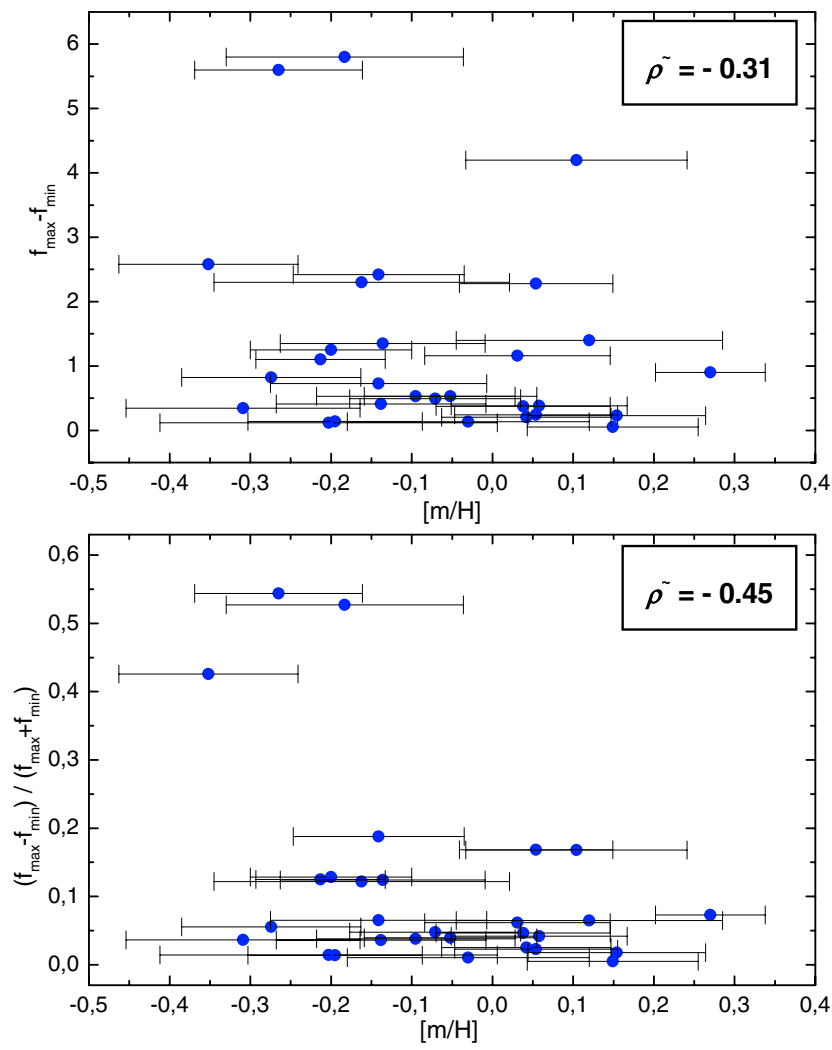

Fig. 3. The range of observed frequencies in $\beta$ Cep stars as a function of $[\mathrm{m} / \mathrm{H}]$. The bottom panel is for the frequency range normalized by the sum of the lowest and highest frequency. The values of weighted correlation coefficient, $\tilde{\rho}$, are given in the right-upper corners.

size of random samples. The null hypothesis $H_{0}: \mu_{X}=\mu_{Y}$ was rejected in favor of $H_{1}: \mu_{X} \neq \mu_{Y}$, as well as in favor of $H_{2}$ : $\mu_{X}<\mu_{Y}$.

The higher value of the mean metal abundance in the case of multiperiodic $\beta$ Cep stars is understandable in the framework of the linear non-adiabatic theory, which predicts more unstable frequencies for higher metal abundance $Z$. Therefore, we tried to find a dependence between the range of observed frequencies and the metal abundance. We cannot draw conclusions about any correlation between these two parameters because the value of the weighted correlation coefficient $\tilde{\rho}$ is -0.45 . In fact, we should not expect more, as the number of detected modes is much lower than theory predicts. We have to remember also that the values of $[\mathrm{m} / \mathrm{H}]$ give information mainly about photospheric metal abundances. Moreover, we do not take into account phenomena like diffusion, element mixing etc.

To improve this analysis we need determinations of the $[\mathrm{m} / \mathrm{H}]$ parameter for a larger sample of $\beta$ Cep stars. Moreover, a high accuracy in the period search analysis is needed to estimate better the range of the excited modes in a given star. Here put our hope on the next multi-site and multi technique campaigns, as well as on space missions. Finally, the analysis of the chemical composition of $\beta$ Cep variables from high resolution spectra would be very helpful.

These results require further studies and must be treated with caution. Firstly, they were obtained for small samples of stars, and secondly, we do not know how many modes of the excited ones have been already identified. Our aim was to show some properties that could be extracted from such determinations of the metal abundance parameter $[\mathrm{m} / \mathrm{H}]$ for $\beta$ Cep variables. Having in mind that from a theoretical point of view all $\beta$ Cep stars should be multiperiodic ones, the metallicity dichotomy we obtained is rather a qualitative confirmation of the dependence of the pulsation instability in these stars on the heavy element abundance.

Acknowledgements. We thank Wojtek Dziembowski and Alosha Pamyatnykh for the use of the Warsaw-New Jersey evolutionary code and the non-adiabatic pulsation code.

\section{References}

Aerts, C., De Cat, P., Handler, G., et al. 2004, MNRAS, 347, 463

Bevington, P. R. 1969, in Data Reduction and Error Analysis for the Physical Science (New York: McGraw-Hill)

Daszyńska, J. 2001, Ph.D. Thesis, Wrocław University, Poland

Daszyńska, J., Niemczura E., \& Cugier, H. 2003, Adv. Space Res., 31, 387

Dziembowski, W. A. 1977, Acta Astron., 27, 203

Dziembowski, W. A., \& Pamyatnykh, A. A. 1993, MNRAS, 262, 204

Gautschy, A., \& Saio, H. 1993, MNRAS, 262, 213

Handler, G., Shobbrook, R. R., Jerzykiewicz, M., et al. 2004, MNRAS, 347, 454

Kiriakidis, M., El Eid, M. F., \& Glatzel, W. 1993, MNRAS, 255, 1P

Kurucz, R. L. 1996, CD-ROM Nos. 13 and 19

Moskalik, P., \& Dziembowski, W. A. 1992, A\&A, 256, L5

Niemczura, E. 2003, A\&A, 404, 689

Niemczura, E., \& Daszyńska-Daszkiewicz, J. 2005, A\&A, 433, 659, Paper I

Pamyatnykh, A. A. 1999, Acta Astron., 49, 119 